\title{
Dendrite-Derived Supernumerary Axons on Adult Axotomized Motor Neurons Possess Proteins That Are Essential for the Initiation and Propagation of Action Potentials and Synaptic Vesicle Release
}

\author{
Claire F. Meehan, ${ }^{1}$ Victoria E. MacDermid, ${ }^{2}$ Steven J. Montague, ${ }^{2}$ Monica Neuber-Hess, ${ }^{2}$ and P. Ken Rose ${ }^{2}$ \\ ${ }^{1}$ Department of Neuroscience and Pharmacology, Panum Institute, University of Copenhagen, DK-2200 Copenhagen N, Denmark, and ${ }^{2}$ Canadian Institutes \\ of Health Research Group in Sensory-Motor Systems, Department of Physiology, Centre for Neuroscience, Queen's University, Kingston, Ontario K7L 3N6, \\ Canada
}

\begin{abstract}
Axotomy can trigger profound alterations in the neuronal polarity of adult neurons in vivo. This can manifest itself in the development of new axon-like processes emanating from the tips of distal dendrites. Previously, these processes have been defined as axonal based on their axonal morphology. This study extends this definition to determine whether, more importantly, these processes possess the prerequisite molecular machinery to function as axons. Using a combination of intracellular labeling and immunohistochemistry, we demonstrate that the distribution of voltage-gated sodium channels on these processes matches the arrangement of these channels that is necessary for the initiation and conduction of action potentials. At terminal bouton-like structures they possess key proteins necessary for the release of synaptic vesicles (SV2 and synaptophysin). Thus, axon-like processes emanating from the tips of distal dendrites represent a rearrangement of neuronal polarity whereby axotomized neurons can develop additional functional axons in vivo.
\end{abstract}

\section{Introduction}

Neurons are polarized structures with multiple dendrites integrating synaptic signals and, usually, a single axon transmitting action potentials triggering the release of neurotransmitters. In vitro manipulations of intracellular signaling cascades responsible for the development/maintenance of neuronal polarity result in single neurons with multiple axons (Inagaki et al., 2001; Jiang et al., 2005; Yoshimura et al., 2005; Witte et al., 2008). After the establishment of neuronal polarity, these manipulations result in the conversion of preexisting dendrites to axons. Thus, neuronal polarity is not fixed. Instead, it appears to be actively maintained by intracellular signal cascades that suppress a default mechanism that would otherwise convert dendrites to axons.

There is considerable evidence that proximal axotomy may trigger a similar reorganization of neuronal polarity both in vitro

Received 0ct. 13, 2010; revised Feb. 9, 2011; accepted March 12, 2011.

Author contributions: C.F.M. and P.K.R. designed research; C.F.M., V.E.M., S.J.M., M.N.-H., and P.K.R. performed research; C.F.M., V.E.M., and M.N.-H. analyzed data; C.F.M. and P.K.R. wrote the paper.

This work was supported by the Canadian Institutes for Health Research (Grants MOP-79299 and MOP-37765). We thank Dr. Keith Brunt for performing the Western blots and Rebecca Cranham for technical assistance. We thank Stacey Armstrong for the reconstruction in supplemental Figure S1 (available at www.jneurosci.org as supplemental material). The SV2 monoclonal antibody was developed by Kathleen M. Buckley and was obtained from the Developmental Studies Hybridoma Bank developed under the auspices of the National Institute of Child Health and Human Development and maintained by the University of lowa, Department of Biological Sciences, lowa City, IA 522422.

Correspondence should be addressed to Claire F. Meehan, University of Copenhagen, Department of Neuroscience and Pharmacology, Panum Institute, Building 18.5, Blegdamsvej 3, 2200 Copenhagen N, Denmark. E-mail: claire@sund.ku.dk.

DOI:10.1523/JNEUROSCI.5377-10.2011

Copyright $\odot 2011$ the authors $\quad 0270-6474 / 11 / 316732-09 \$ 15.00 / 0$
(Bradke and Dotti, 2000; Gomis-Rüth et al., 2008) and in vivo (Lindå et al., 1985, 1992; Havton and Kellerth, 1987; Rose and Odlozinski, 1998; Rose et al., 2001; MacDermid et al., 2002, 2004). This reorganization is manifested in the emergence of axon-like processes (ALPs) from the terminals of distal dendrites (Lindå et al., 1985, 1992; Rose et al., 2001; MacDermid et al., 2002, 2004; Gomis-Rüth et al., 2008). With time, these ALPs become myelinated (Lindå et al., 1992; Rose et al., 2001) and develop fine right-angled branches with bouton-like swellings, and the dendrites that give rise to these ALPs undergo a process of apparent axonification (MacDermid et al., 2004). ALPs lack dendritic cytoskeletal proteins and initially express the axonal growth proteins (Rose et al., 2001; MacDermid et al., 2002, 2004). Thus, the ALPs meet some of the morphological and molecular criteria of axons; however, it is unknown whether they have the functional capacity of an axon. Our goal was therefore to determine whether ALPs possess the prerequisite molecular machinery for action potential initiation and conduction and the release of neurotransmitters.

Action potentials are initiated at the initial segment of an axon (Kole et al., 2008) by high-density clusters of the voltage-gated sodium channels Nav1.1 and Nav1.6 (Van Wart et al., 2007) and, during development, Nav1.2 (Boiko et al., 2001). Similarly, in developing and adult unmyelinated axons, action potentials are conducted along the axon by Nav1.2 channels and in adult myelinated axons by Nav1.6 (Boiko et al., 2001, 2003; Kaplan et al., 2001; Trimmer and Rhodes, 2004). The release of synaptic vesicles is dependent on a number of proteins. Synaptophysin and SV2 are two integral membrane glycoproteins playing key roles in 
the exocytosis of synaptic vesicles and are widely used as synaptic markers (Finley et al., 1996; Valtorta et al., 2004).

In the present experiments we demonstrate that dendritederived ALPs on axotomized neurons in adult animals in vivo possess voltage-gated sodium channels, SV2 and synaptophysin, in discrete regions of the ALPs. These regions correspond precisely to the known distribution of these proteins on axons that initiate and conduct action potentials and release neurotransmitters. Thus, they possess the prerequisite molecular machinery to function as axons.

\section{Materials and Methods}

Axotomy surgery

All experimental protocols were approved by Queen's University Animal Care Committee and were consistent with guidelines established by the Canadian Council of Animal Care. Ten adult cats of both sexes (Harlan) were premedicated with hydromorphone $(0.05 \mathrm{mg} / \mathrm{kg})$, glycopyrrolate $(0.001 \mathrm{mg} / \mathrm{kg})$, and acepromazine $(0.13 \mathrm{mg} / \mathrm{kg})$. Anesthesia was then induced with ketamine $(0.25 \mathrm{mg} / \mathrm{kg})$ and midazolam $(0.31 \mathrm{mg} / \mathrm{kg})$ and maintained with isoflurane (2-3\%). Under aseptic surgical conditions, a midline incision was made and the trapezius and splenius neck muscles were retracted to expose the $\mathrm{C} 2$ and $\mathrm{C} 3$ nerves innervating the biventer cervicis and complexus (BCCM) neck muscles. Both the $\mathrm{C} 2$ and $\mathrm{C} 3$ BCCM nerves were dissected away from the muscle, ligated in two places, and then cut between the two ties. The proximal stumps were crushed three times, pulled into a small-diameter Gortex (W.L. Gore and Associates) sleeve, and secured with silk ligatures placed at either end of the sleeve. A second, larger Gortex sleeve was then placed over the first. This second sleeve was longer and was pulled as far proximally as possible. The second sleeve was designed to prevent the escape of regenerating axons from the first sleeve as was sometimes observed in previous experiments after post-axotomy periods of 35 weeks (MacDermid et al., 2004). The muscle layers and skin were sutured with Vicryl, and an analgesic, buprenorphine $(0.01 \mathrm{mg} / \mathrm{kg})$, was administered as well as an antibiotic, Tribrissen $(24 \%, 0.13 \mathrm{ml} / \mathrm{kg})$. Further doses of buprenorphine were administered as required, and a nonsteroidal anti-inflammatory (Metacam, 3 drops daily) and Tribrissen (dosage as above) were administered daily for $6 \mathrm{~d}$. The only postoperative complications were bald patches on the skin, rostral to the injury site, caused by scratching. Treatments with Panalog topical ointment and a nonsteroidal anti-inflammatory (Metacam, 3 drops daily) usually resolved these bald patches.

\section{Identification and labeling of neurons}

After survival times of 8-12 weeks ( 4 cats) or 33-38 weeks (6 cats), the animals were premedicated with a mixture of butorphanol $(0.2 \mathrm{mg} / \mathrm{kg}$; Torbugesic; Pfizer), acepromazine $(0.05 \mathrm{mg} / \mathrm{kg}$; Atravet; Ayerst Veterinary Laboratories), and glycopyrrolate $(0.01 \mathrm{mg} / \mathrm{kg}$; Sandoz) given subcutaneously. Anesthesia was induced with ketamine $(6.25 \mathrm{mg} / \mathrm{kg}$; Vetalar; Bioniche Animal Health Canada Inc.) and midazolam (0.15 mg/ $\mathrm{kg}$; Sandoz) administered intravenously. Deep anesthesia was maintained with supplementary doses of sodium pentobarbital (Ceva Santé Animale; $2.5-5 \mathrm{mg} / \mathrm{kg}$ ). The proximal stumps of the BCCM nerves, still encased in their Gortex sleeves, were dissected from connective tissue, and bipolar stimulating electrodes were placed immediately proximal to the sleeve. A laminectomy was performed to access the C2 and C3 spinal segments. Axotomized motor neurons were identified by intracellular recordings of antidromic action potentials following stimulation of the proximal stump. These cells were iontophoretically stained with $12 \%$ Neurobiotin (Vector Laboratories) for 16-55 nA-min. Filled cells were separated by at least $5 \mathrm{~mm}$ to minimize overlap of the dendritic trees of adjacent cells.

Animals were given 25,000 IU of heparinized saline and killed with an overdose of sodium pentobarbitone. The animal was immediately perfused, via the ascending aorta, with saline at room temperature followed by $4 \%$ paraformaldehyde $\left(\right.$ at $4^{\circ} \mathrm{C}$ ) at a drip rate of $3 \mathrm{drops} / \mathrm{s}$ for $10 \mathrm{~min}$, if the antibodies to be used were fixation dependent or, otherwise, for 15 $\mathrm{min}$. Tissue was also collected from two animals that were not injured. In one of these animals, an intact BCCM motor neuron was intracellularly stained as described above.

\section{Histology}

The spinal cord segments containing labeled cells (C2 and C3) were stored overnight in buffer with $30 \%$ sucrose. Tissue was then postfixed for $1 \mathrm{~h}$ before cutting on a freezing microtome in the horizontal plane into $50 \mu \mathrm{m}$ serial sections. Tissue was incubated in sodium borohydride for $20 \mathrm{~min}$, followed by $3 \mathrm{~h}$ in streptavidin conjugated to Alexa 488 (1:100; Invitrogen) in phosphate buffer to visualize the Neurobiotin. Sections were mounted in serial order on gelatin-coated slides and coverslipped with Vectashield (Vector Laboratories).

All sections containing intracellularly stained processes were systematically examined. ALPs were identified using one or more of the following criteria (based on the study by MacDermid et al., 2004). They followed a tortuous trajectory and formed either simple or complex arborizations that consisted of fine branches that emerged at right angles and had several bouton-like swellings. The parent process could be followed to its origin at the soma or proximal dendrite. The original axon was always identified (by its trajectory toward the ventral roots) and clearly arose from a different site on the soma.

Tissue sections containing the soma and the immediately adjacent sections were processed immunohistochemically for sodium channels using a pan-sodium channel antibody to label initial segments. Tissue sections containing thick branches of ALPs that did not taper and matched the staining characteristics of myelinated "dendrites" (indicated by the disappearance and reappearance of the process as it traveled through the section) (Cullheim and Kellerth, 1978; Rose and Odlozinski, 1998; Rose et al., 2001) were processed immunohistochemically with either the pan-sodium channel antibody or an antibody against Nav1.2. Other tissue sections that contained ALPs that gave off fine branches that arborized extensively and had structures resembling boutons were processed immunohistochemically for SV2 and/or synaptophysin.

Colocalization was initially analyzed using an epifluorescent microscope and then was confirmed using a confocal microscope. Partial reconstructions were performed using the Neurolucida tracing system (MicroBrightField). Further colocalization analysis was performed using NIH ImageJ and Photoshop (Adobe). Images were optimized using the gain and offset on the confocal microscope. The only post-acquisition manipulation to images was to enhance the contrast of images that contained the blue fluorochrome, and this was performed using Adobe Photoshop (the confocal microscope was not equipped with the appropriate lasers for this wavelength).

\section{Immunohistochemistry}

Pan-sodium channel immunohistochemistry. Labeling of clusters of sodium channels was achieved using a pan-sodium channel antibody (Sigma, lot number 045K6083) recognizing all types of mammalian sodium channels. Labeling using this antibody was highly dependent on the level of fixation of the tissue and required a perfusion time of $10 \mathrm{~min}$ or less, rendering the tissue fragile and prone to fragmentation. Before processing tissue sections containing the process of interest, tissue from the same experiment was processed using this antibody to confirm that the fixation level was optimal to label initial segments.

Tissue was incubated for $2 \mathrm{~h}$ in PBS with 5\% goat serum with 3\% Triton X-100. The pan-Na channel antibody was diluted to a working concentration of 1:500 and spun on a microcentrifuge for $10 \mathrm{~min}$. The tissue was incubated for $24 \mathrm{~h}$ at $4^{\circ} \mathrm{C}$ and was followed by a second incubation with a goat-anti-mouse secondary antibody conjugated to Cy3 (1:1000; Jackson ImmunoResearch Laboratories) for $24 \mathrm{~h}$ at room temperature.

In our tissue, the pan-sodium channel antibody only labeled structures that resembled nodes of Ranvier and axonal initial segments, locations known to possess high densities of sodium channels (Shrager, 1987; Hedstrom and Rasband, 2006; Kole et al., 2008). The same structures were labeled in sections collected from the spinal cord of the rat tissue using an antibody directed against the Nav1.6 protein (NeuroMabs, 1:100, UC Davis/NIH NeuroMab Facility; protocol as for pan-sodium channel antibody), the sodium channel known to be clustered at nodes of Ranvier and initial segments (data not shown). Thus, the pan-sodium channel antibody, in our hands, only labeled regions with a high density of sodium channels. 
Labeling of presumed initial segments with the pan-sodium channel antibody was confirmed using the tissue from uninjured animals. Double immunohistochemical labeling was also performed using the pan-sodium channel antibody (1:500 protocol as before) along with an antibody raised against choline acetyltransferase (ChAT) (Millipore Bioscience Research Reagents, 1:100) which was visualized with the green fluorochrome Alexa 488 conjugated to an anti-rabbit secondary antibody (1:100, Jackson ImmunoResearch Laboratories).

Nav1.2 immunohistochemistry. Tissue sections containing the ALP and its fine branches were processed using an antibody directed against the amino acid residues $467-485$ of the Nav1.2 protein (Millipore Bioscience Research Reagents; lot number 0607036525). Before blocking, antigen retrieval was performed using a high- $\mathrm{pH}$, high-temperature sodium citrate buffer. After cooling to room temperature and rinsing, the tissue was incubated for $2 \mathrm{~h}$ in 5\% goat serum in PBS with 3\% Triton X-100. The primary antibody was diluted to a final working concentration of $1: 250$ in PBS with $0.6 \%$ Triton X-100 with $5 \%$ goat serum and spun on a minicentrifuge for $10 \mathrm{~min}$. The tissue was incubated in the primary antibody for $24 \mathrm{~h}$. A secondary antibody, goat-anti- mouse, conjugated to Cy-3 (Jackson ImmunoResearch Laboratories) was diluted to a final working concentration of 1:500 in PBS with $0.3 \%$ Triton X-100. Tissue was incubated with the secondary antibody for $2 \mathrm{~h}$ at room temperature. Preincubation of the primary antibody with the purified peptide (1:10 for $2 \mathrm{~h}$ ) abolished all labeling. This suggests that this antibody is specific for this sequence and, therefore, for Nav1.2. However, the sequence used to generate the Nav1.2 antibody shares a high degree of homology with the Nav1.3 protein (12 of 15 aa residues). Western blots on cat tissue using the Nav1.2 antibody produced a band at the correct molecular weight for Nav1.2 $(\sim 270 \mathrm{kDa})$ along with another band at $50 \mathrm{kDa}$. Although BLAST (basic local alignment search tool) searches revealed no other protein with high homology to this sequence, Nav1.2 and Nav1.3 have similar molecular weights. We therefore examined tissue stained with an antibody directed specifically at the Nav1.3 protein (1:100, Alomone Laboratories; protocol as for anti-Nav1.2 antibody). Since the pattern of staining did not match that seen with the Nav1.2 antibody, we concluded that the Nav1.2 antibody does not bind to Nav1.3.

SV2/synaptophysin immunohistochemistry. SV2 was labeled using a mouse-anti-SV2 antibody from the Developmental Studies Hybridoma Bank (University of Iowa, Iowa City, IA). Tissue was permeabilized by incubation in a blocking solution of $5 \%$ goat serum with $1 \%$ Triton X-100 in PBS for $24 \mathrm{~h}$, followed by a second incubation for $48 \mathrm{~h}$ in the primary antibody (1:50) diluted in PBS with $1 \%$ Triton X-100 and saponin. Tissue was incubated for $2 \mathrm{~h}$ with the secondary antibody, goat-anti-mouse, conjugated to CY-3 (1:500, Jackson ImmunoResearch Laboratories) diluted in PBS with 6\% Triton X-100. Processing using the synaptophysin antibody (1:100, Zymed) was performed using the same protocol as for the SV2 antibody with a goat, anti-rabbit secondary antibody conjugated to aminomethylcoumarin acetate (Jackson ImmunoResearch Laboratories). Tissue labeled with this blue fluorochrome was mounted using Citifluor mounting medium.

When double labeling was performed using two of any of the different primary antibodies, the tissue was sequentially incubated in each secondary antibody. Controls for cross-reactivity were performed by incubating tissue in each primary antibody alone and then with the secondary antibody for the other primary antibodies, and also with just one primary antibody with both secondary antibodies. No cross-reactivity was observed.

\section{Electron microscopy}

Electron microscopy was performed on two cells. In this case the animal was perfused with $1 \%$ paraformaldehyde and $2.5 \%$ glutaraldehyde, and $0.01 \% \mathrm{CaCl}$ in $0.1 \mathrm{M}$ cacodylate buffer, $\mathrm{pH} 7.4$, and the Neurobiotin was visualized with an avidin-biotin-peroxidase complex and a diaminobenzidine reaction. Embedding, sectioning, and postembedding procedures were performed as described previously (Rose and Neuber-Hess, 1991).

\section{Results}

Data were collected from 21 proximally axotomized neurons that previously innervated the BCCM neck muscles of adult cats at 33-38 weeks post-axotomy. In all of these cells, the Neurobiotin staining extended to the ends of the most distal branches of the dendritic tree and axon collaterals.

Sixteen of the neurons possessed ALPs in addition to their original axons. The original axons were clearly identifiable by their trajectory toward the ventral roots, and the branch points of their fine collateral branches (which persisted with bouton-like structures) could be clearly seen, allowing both to be distinguished from the ALPs. In total, 22 ALPs were identified on these cells. The most proximal (to the soma) parts of ALPs were relatively simple (taking a direct trajectory path with little or no branching) and very fine $(<1 \mu \mathrm{m})$ compared with proximal dendrites on the same cell (Fig. 1A). The most proximal parts were also noticeably thinner than that of the original axons of the neurons. These processes, however, became thicker $(>2 \mu \mathrm{m})$ distally and traveled for considerable distances beyond that normally observed for dendrites $(>2 \mathrm{~mm})$. Distally they remained thick, did not taper, followed meandering trajectories, and possessed fine right-angled branches, which arborized extensively (Fig. $1 B$, arrows). As far as the Neurobiotin labeling could be followed, none of the ALPs took a trajectory toward the ventral roots, the route normally taken by the axons of spinal motor neurons. Instead, ALPs were widely distributed throughout the ventral horn (in both the gray and white matter) and the intermediate zone. The fine processes resembled axon collateral branches, and these branches possessed both en-passant and terminal swellings. The branches had the tendency to arise at considerable distances from the cell body (at least $1 \mathrm{~mm}$ ) and appeared to have no one preferred region of termination, occupying regions in the gray matter of the ventral horn and intermediate zone (including lamina $\mathrm{X}$ ), as well as the ventromedial, ventrolateral, and ventral white matter and ventral commissure.

\section{At 33-38 weeks post-axotomy, ALPs have initial segments as defined by a clustering of voltage-gated sodium channels} Immunohistochemical labeling of control tissue using a pansodium channel antibody (Sigma) revealed clusters of sodium channels on structures that resembled initial segments and nodes of Ranvier (small dots had dimensions that are typical of nodes of Ranvier, and thicker, longer regions of immunoreactivity matched the size and known distribution of sodium channels on the initial segments of neurons). ChAT labeling (data not shown) confirmed that only one cluster of sodium channels resembling an initial segment was seen per motor neuron (based on a sample of $>50$ cells). On uninjured BCCM motor neurons, only one initial segment was labeled per cell.

The conditions under which this antibody will work are restricted (see Materials and Methods). As a consequence, only two of the five 33- to 38-week experiments were processed using this antibody. Four examples (from three cells) where the most proximal part of an ALP was captured in the same 50- $\mu$ m-thick tissue section as the cell body were selected and tested. All four processes could be followed distally, through serial tissue sections, where they became thicker $(>2 \mu \mathrm{m})$ and had branches with bouton-like swellings. A discrete cluster of sodium channels colocalized with all four ALPs close to the soma-ALP junction, similar to the location of sodium channels on the initial segment of the axons of intact cells (Fig. $2 A, B$ ). No labeling was detected on processes that resembled dendrites identified by their distinc- 
A

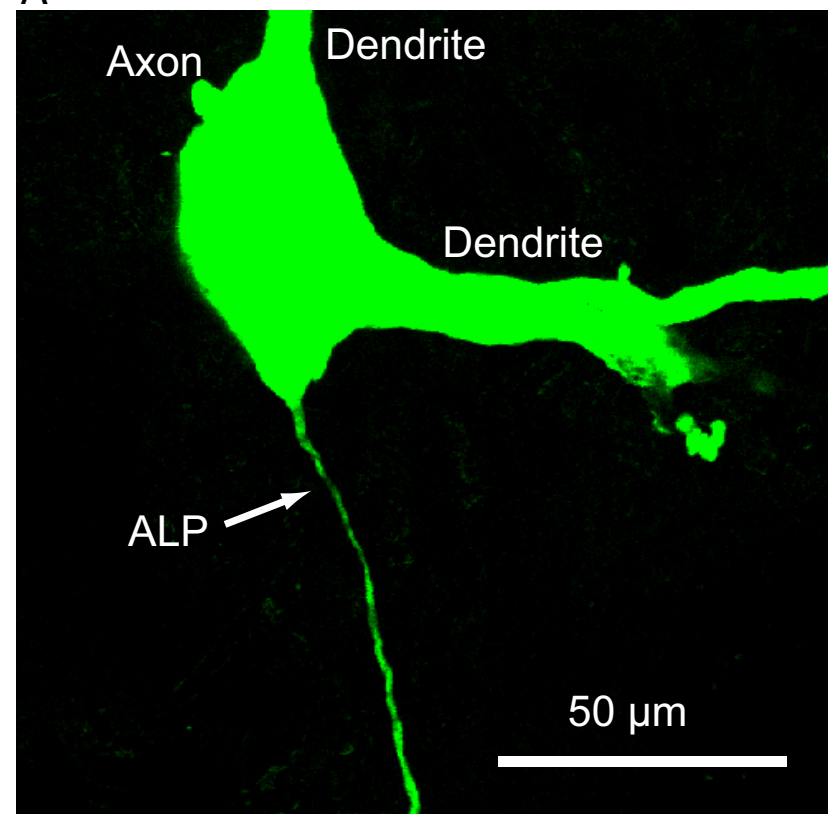

B

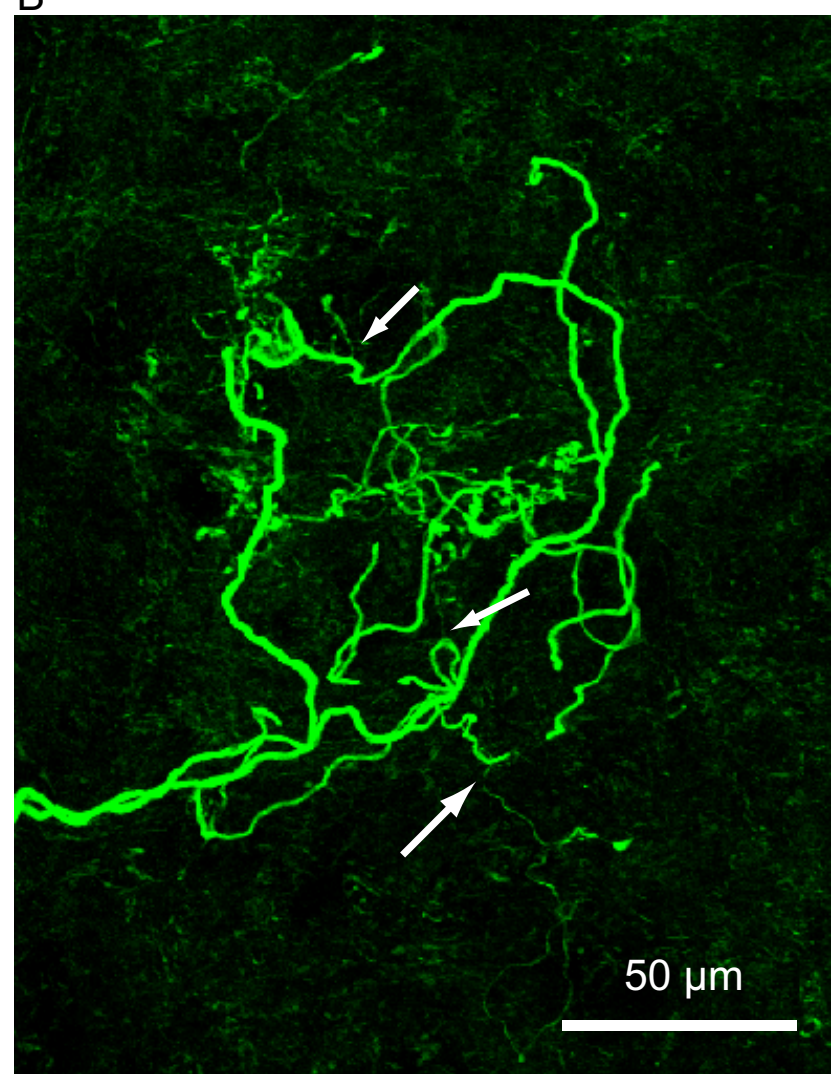

Figure 1. Morphological features of ALPs. A, Confocal images based on a stack of $31,1-\mu \mathrm{m}$ thick, optical sections of the cell body of an axotomized neuron. The proximal part of an ALP is shown (arrow). $\boldsymbol{B}$, Another confocal stack of 37, 1- $\mu$ m-thick, optical sections showing the distal portion of an ALP with fine meandering collateral branches (white arrows) branching off of the main ALP at right angles.

tive morphology (for example, tapering and acute angled branching patterns).

It is worth noting that the initial portion of the original axons (identified by its path toward the ventral roots) had a cluster of immunoreactivity for sodium channels consistent with an initial segment, as can be seen in Figure $2 \mathrm{Bi}$. Thus, the initial segment of the original axon appears to be intact, which is consistent with the ability to intracellularly record antidromic action potentials (with both the initial segment and soma-dendritic components) in all these neurons following stimulation of the peripheral stump (the method by which all cells were identified).

At 33-38 weeks post-axotomy, the membranes of ALPs are immunoreactive for the voltage-gated sodium channel Nav1.2, a prerequisite ion channel for the conduction of action potentials in nonmyelinated or partially myelinated axons in the spinal cord.

Clusters of sodium channels resembling mature nodes of Ranvier were not detected on thick ALPs using the pan-sodium channel antibody. Thick ALPs, however, were clearly immunoreactive using an antibody directed against a sequence of amino acids that forms part of the voltage-gated sodium channel Nav1.2 (Fig. 3). Labeling was restricted to the edges of the processes and appeared to surround the Neurobiotin that filled the cells intracellularly, suggesting that the channels are membrane-bound (Fig. $3 B$ ). All 11 ALPs selected from 7 cells displayed immunoreactivity for Nav1.2. This was confirmed in at least two different regions of each ALP (usually on different sections). These 11 ALPs included the 4 examples that had initial segments labeled with the pan-sodium antibody (although the presumed initial segment region was not tested for Nav1.2 immunoreactivity). Nav1.2 immunoreactivity was observed on both proximal and very distal regions of the ALPs. Fine collateral-like processes emanating from these ALPs were also immunoreactive for the Nav1.2 antibody (Fig. 3C).

Nav 1.2 is usually associated with unmyelinated or sparsely myelinated axons (Boiko et al., 2001; Kaplan et al., 2001; Trimmer and Rhodes, 2004). Yet previous studies have reported internode-like regions on ALPs at 8-12 weeks post-axotomy with up to 24 layers of myelin (Rose et al., 2001). We therefore examined the distribution of myelin on 2 ALPs at 33-38 weeks postaxotomy. As in the previous studies, we found a one-to-one correspondence between faintly stained segments of the ALPs seen at the microscopic level and myelinated segments seen at the electron microscopic level (supplemental Fig. S1, available at www.jneurosci.org as supplemental material). The faintly stained regions were most commonly associated with thicker processes and often spanned distances of 50-100 $\mu \mathrm{m}$. Some of these regions were separated by short, intensely stained segments. However, we saw no obvious clustering of Nav1.2 or pan-sodium channel labeling on dendrite-derived supernumerary axons (DDSAs) processed with fluorescent markers (except initial segments), suggesting that nodes of Ranvier have not fully formed at 33-38 weeks post-axotomy.

The original axons of 7 intracellularly labeled axotomized cells (4 cats) were followed through multiple serial sections in the ventral funiculus. All 7 were strongly immunoreactive for Nav 1.2 and were adjacent to other Nav1.2-labeled axons that traveled in discrete bundles toward the ventral root (supplemental Fig. S2, available at www.jneurosci.org as supplemental material). Based on their location and trajectory, these bundles likely originate from other axotomized BCCM motor neurons. The distribution of the Nav1.2 staining on these axons strongly resembled the pattern found on ALPs. This distribution is contrary to what is normally seen on myelinated motor neuron axons, suggesting a possible disruption of nodes of Ranvier in the original axons. 
At 33-38 weeks post-axotomy, boutonlike structures on fine collateral processes from ALPs are immunoreactive for proteins necessary for the release of synaptic vesicles

Examples of bouton-like structures were selected from 10 ALPs ( 9 cells) and were processed immunohistochemically with antibodies raised against the SV2 protein. For each ALP, multiple examples of immunoreactive bouton-like structures were observed. The immunoreactivity was not observed along the length of the processes. Labeling was restricted to bouton-like structures (Fig. 4A,B), both of the terminal and the en passant kind. Bouton-like structures at the distal parts of the branches of the ALP appeared to be more likely to be immunoreactive than en passant swellings at more proximal regions. However, we cannot exclude that this pattern of staining was due to the limited tissue penetration of the antibody. Thus, a quantitative analysis was not possible. Three examples of boutons immunoreactive for SV2 were also processed immunohistochemically with an antibody raised against the synaptophysin protein and were also immunoreactive for synaptophysin, confirming the appropriateness of SV2 as a synaptic marker (Fig. $4 B$ ).

The time course of the arrival of the prerequisite proteins is consistent with the development of the appropriate morphological structures

At 33-38 weeks post-axotomy, every time a structure was identified as a putative initial segment of an ALP or as bouton-like, it was always immunoreactive for the appropriate protein (i.e., pan-sodium channel or SV2/synaptophysin) when tested. The converse is also true; immunoreactive positive regions (for pan-sodium channel, Nav.1.2, or SV2/synaptophysin) of the ALPs were always located in regions consistent with the expected morphological structures (i.e., initial segment, bouton-like swelling). To determine whether the arrival of these proteins coincides with the formation of the appropriate morphological structures, 16 motor neurons (4 cats) were labeled at 8-12 weeks postaxotomy (in 2 cats the neck motor neurons innervating the trapezius muscle were axotomized proximally by transecting the spinal accessory nerve at the $\mathrm{C} 1$ ). All motor neurons had ALPs consistent with those described at similar time points (see MacDermid et al., 2004). In these motor neurons the initial portion of the dendrites giving rise to the ALP did not have a clear cluster of sodium channels as identified with the pan-sodium channel antibody (although the initial segment of the original axon was labeled). This was consistent with their morphology, as the most proximal part of these processes was indistinguishable from primary dendrites at this time point and did not resemble initial segments. Thus, the axon initial segment (AIS) must form on these ALPs somewhere between 8 and 12 and 33 and 38 weeks
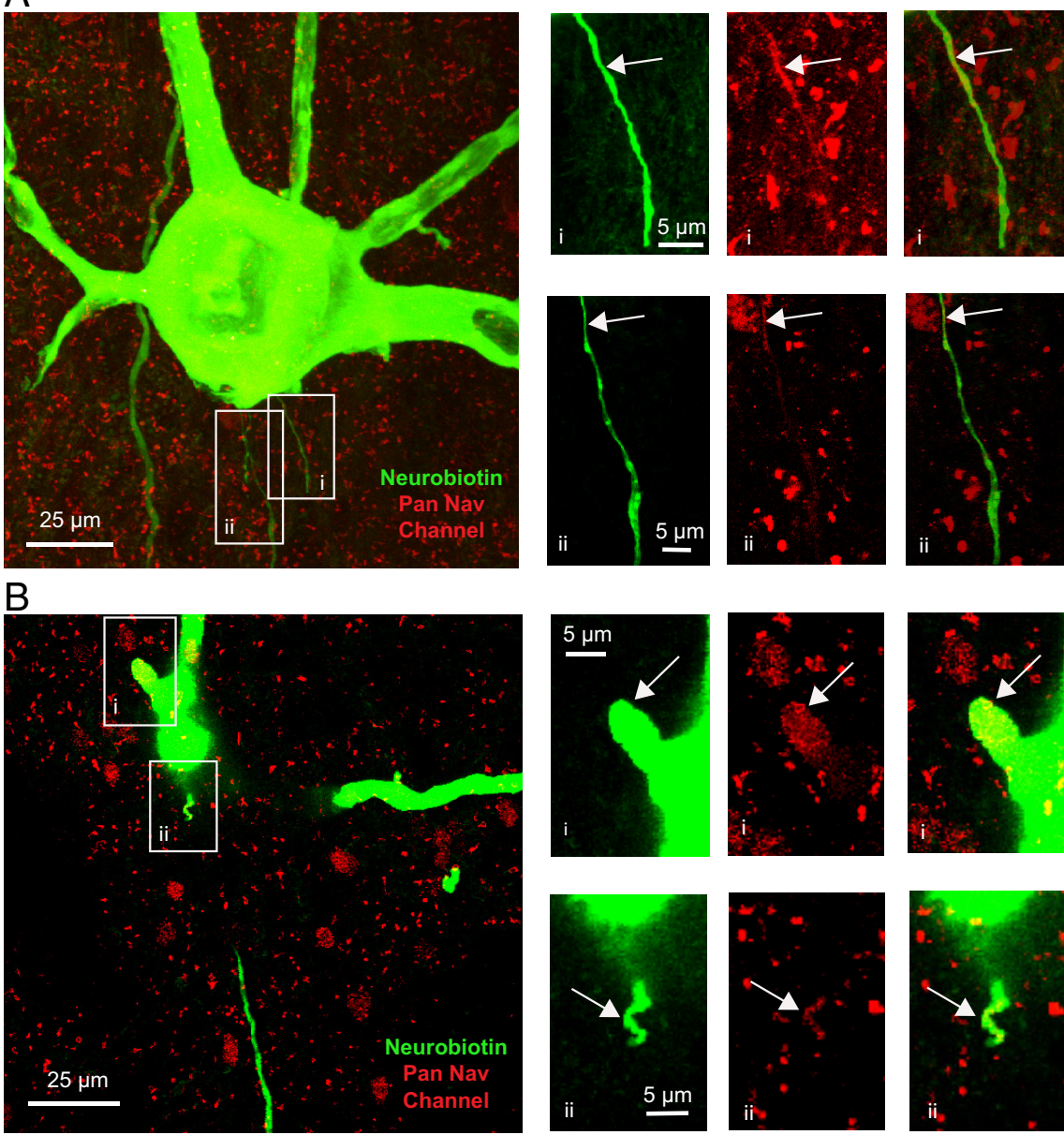

Figure 2. Labeling of initial segments. $\boldsymbol{A}$, Confocal image showing examples of presumed initial segments of two ALPs arising (arrows). Inset $i$, Stack of $6 \times 1-\mu \mathrm{m}$-thick confocal optical slices showing the most proximal part of one ALP from this cell rocess, consistent with that of an initial segment (white arrow). Inset ii, Stack of $3 \times 1$ - $\mu$ m-thick confocal optical slices of with an initial segment on this cell (white arrow). $\boldsymbol{B}$, Confocal image showing another example of a presumed initia channel antibody (confocal stack of $4 \times 1-\mu \mathrm{m}$-thick sections). Inset ii, The most proximal part of the ALP (white arrow) is also immunoreactive for the pan-sodium channel antibody (confocal stack of $4 \times 1-\mu \mathrm{m}$-thick sections).

post-axotomy. We also observed a different distribution of synaptophysin on ALPs that originated from motor neurons at 8-12 weeks post-axotomy. At this stage, ALPs do not have bouton-like structures (MacDermid et al., 2004). Only 3 of 20 ALPs examined were immunoreactive for synaptophysin (supplemental Fig. S3, available at www.jneurosci.org as supplemental material). The immunoreactivity was confined to the terminals or short terminal branches of these ALPs. All of these ALPs formed a small fan-shaped swelling that was uniformly stained with the synaptophysin antibody. Punctate labeling was never observed. The arrival of sodium channels at the initial segment and a punctate distribution of synaptic vesicle proteins at bouton-like structures therefore coincide with the development of the appropriate morphological structures.

\section{Discussion}

Previous in vivo studies of ALPs in the adult mammalian nervous system have described these processes in terms of their 


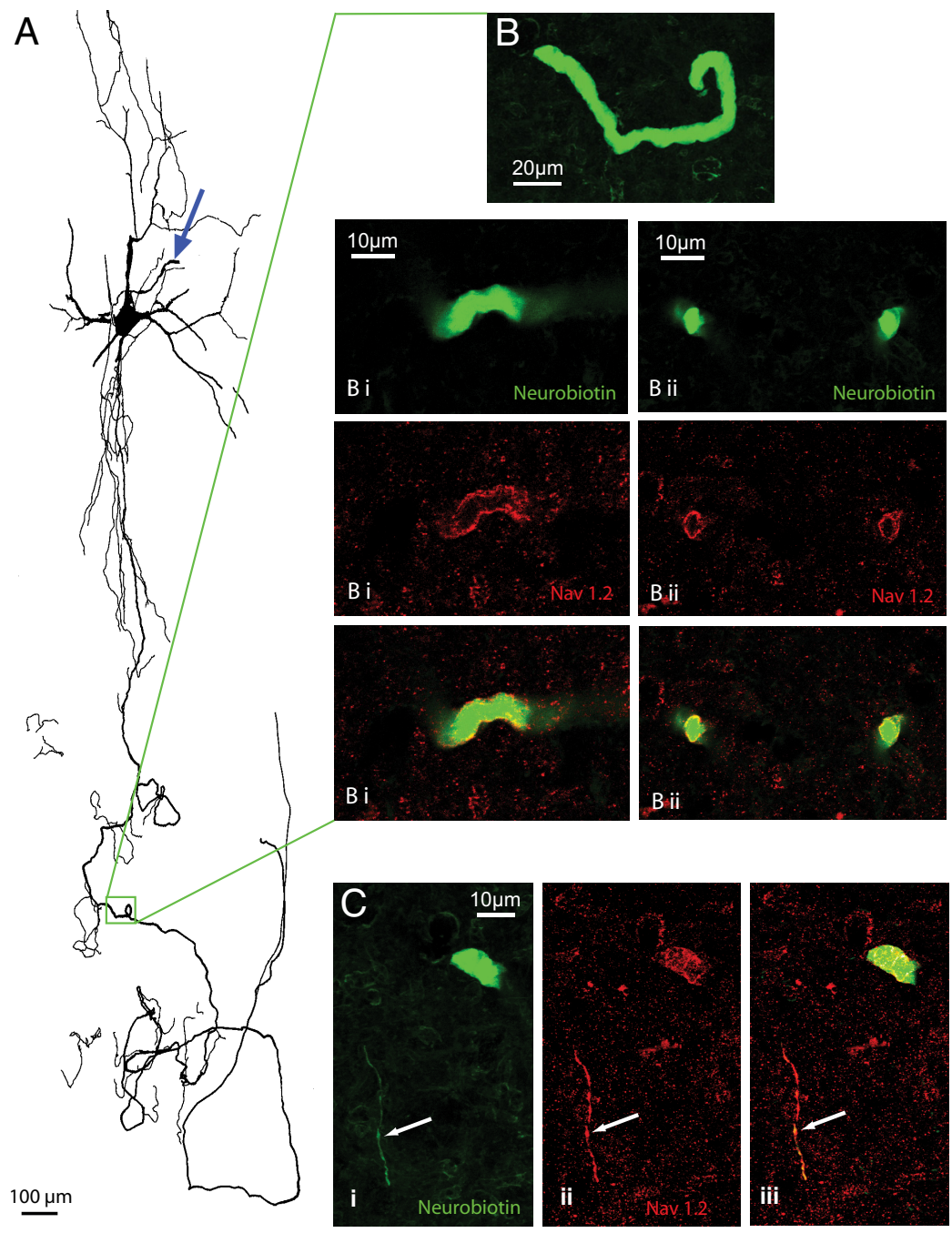

Figure 3. Nav1.2 on new dendrite-derived axon-like processes of an axotomized motor neuron. $A$, Partial reconstruction of an intracellularly labeled axotomized motor neuron showing a thick meandering ALP with fine collaterals branches (blue arrow indicates the proximal part of the original axon which was identified by its trajectory toward the ventral roots). $\boldsymbol{B}$, Confocal stack (from $29 \times 1-\mu \mathrm{m}$-thick optical sections) of a distal region of the ALP (indicated by the green box in Fig. $3 A$ ). Inset $\boldsymbol{i}$, Single $1-\mu \mathrm{m}$-thick confocal images used to generate the stacked image (green) with immunohistochemical labeling of the voltage-gated sodium channel Nav1.2 (red) showing the Nav1.2 labeling on the edge of the process. Inset ii, Another single 1- $\mu \mathrm{m}$-thick confocal image of the above ALP (green) with immunohistochemical labeling of the voltagegated sodium channel Nav1.2 (red) showing the Nav1.2 labeling in and surrounding the process. C, Fine collateral-like process (white arrows) from the ALP labeled with Neurobiotin (i) and Nav1.2 labeling in red (ii), which colocalize (iii).

similarity to the cytoskeletal and anatomical features of axons (Lindå et al., 1992; Rose et al., 2001; MacDermid et al., 2004). We now demonstrate that ALPs that emerge from the distal dendrites of axotomized neurons also possess the prerequisite molecular machinery for action potential initiation, action potential propagation, and the release of synaptic vesicles. All of these features are functional hallmarks of axons. Data from a shorter post-axotomy interval confirms that the arrival of these proteins coincides with the formation of the appropriate morphological structures.

\section{Changes in polarity due to axotomy depend on the} experimental model

Changes in neuronal polarity following axotomy have also been previously demonstrated in both culture and slice preparations (Bradke and Dotti, 2000; Hayashi et al., 2002; Gomis-Rüth et al., 2008). Despite the fact that proximal axotomy leads to the con- version of dendrites into axons regardless of the type of experimental preparation, in vivo or in vitro, or the age of the neurons, there are major differences in the time course of the conversion (being considerably slower in vivo), the conditions that lead to the conversion (requiring a less proximal axotomy in vivo), and the consequences of axotomy to the original axon (which is lost in vitro but persists in vivo). Thus, in vivo, the neuron gains one or more new axons leading to a multi-axon neuron.

The in vitro studies have been performed using embryonic or early postnatal neurons (Bradke and Dotti, 2000; Hayashi et al., 2002; Gomis-Rüth et al., 2008). The finding that axotomy on the adult nervous system in vivo leads to changes in polarity that mimic some, but not all, of the changes seen in in vitro studies therefore suggests that axotomydriven changes in neuronal polarity are determined, in part, by the maturity of the nervous system. If factors maintaining neuronal polarity during development are the same in the adult, then the distribution and timing of changes in the expression of these factors must be appropriately adjusted in the adult nervous system. It is also plausible, however, that the underlying mechanisms responsible for the regulation of polarity following axotomy may be fundamentally different in the adult nervous system, in vivo.

\section{Nomenclature: dendroaxons or supernumerary axons?}

In the current experiments all ALPs had proximal processes morphologically resembling axons rather than dendrites. At post-axotomy intervals of $<12$ weeks, all ALPs emerge from the tips of distal dendrites (MacDermid et al., 2002). This suggests that dendrites giving rise to ALPs lose their dendritic identity over the course of $\sim 20$ weeks and are transformed into the proximal segments of the new axon. This is consistent with the gradual distal to proximal retrograde loss of the dendritic cytoskeletal protein MAP2a/b from the dendrite (Hayashi et al., 2002; MacDermid et al., 2004; Takahashi et al., 2007). We now demonstrate the axonification of the original dendrite giving rise to the ALP at a functional level as indicated by the presence of an initial segment (defined by a clustering of sodium channels). This suggests that the term dendroaxon (Lindå et al., 1985) that has been used to define these processes is only appropriate during the early stages of the development of ALPs and represents a transitory stage. At longer postaxotomy intervals, ALPs are largely indistinguishable from additional axons which sometimes emanate from cell bodies following distal axotomies (Havton and Kellerth, 1987). These axons were called "supernumerary axons." In keeping with this nomenclature, ALPs are best described as dendritederived supernumerary axons (DDSAs). 
Functional capacity and density/distribution of proteins essential for the initiation and propagation of action potentials and synaptic vesicle release

While the presence of voltage-gated sodium channels is a prerequisite for the initiation and conduction of action potentials, this function depends upon the absolute density of channels clustered at crucial locations. While we have no quantitative data to address this issue, the intensity/density of labeling of sodium channels that we observed on the DDSAs matched what we have observed in our laboratory on injured axons, which conducted antidromic action potentials, and on axon collaterals in the gray matter of uninjured neurons (C. F. Meehan and P. K. Rose, unpublished observation), suggesting that the density of sodium channels on DDSAs may be sufficient to conduct action potentials. The dispersed distribution of Nav1.2 along the DDSA is typical of unmyelinated or partially myelinated axons (Westenbroek et al., 1992; Boiko et al., 2001; Craner et al., 2003). While sparse regions of myelination on DDSAs are present at earlier post-axotomy time intervals (Lindå et al., 1992; Rose et al., 2001), the extent of myelination of DDSAs at 33-38 weeks postaxotomy is unknown. The fact that Nav 1.2 channels are not confined to discrete zones at 33-38 weeks post-axotomy suggests that myelination is incomplete. While this may affect the conduction velocity of action potentials, it would not impair the ability of DDSAs to conduct action potentials, as conduction in unmyelinated axons is based solely on dispersed Nav1.2 channels.

To what extent does the labeling of boutons with the SV2/synaptophysin antibodies represent mature functional synapses? The uniform labeling of distal structures at short intervals post-axotomy are consistent with that seen on growth cones of developing axons and in contrast with the punctate labeling observed in mature, functional, synapses (Fletcher et al., 1991). At 33-38 weeks post-axotomy, SV2 and synaptophysin immunoreactivity on DDSAs is punctate and restricted to terminal and en passant bouton-like structures. The time-dependent redistribution of these proteins is consistent with the formation of functional synapses seen during development (Fletcher et al., 1991; Quinn et al., 2003), providing strong support that SV2/ synaptophysin-labeled boutons on DDSAs are functional synapses.

\section{Possible functions of DDSAs}

It has been suggested that the development of DDSAs on axotomized cells may be a mechanism by which the neuron attempts to replace the original axon (MacDermid et al., 2004; Gomis-Rüth et Adobe Photoshop software.
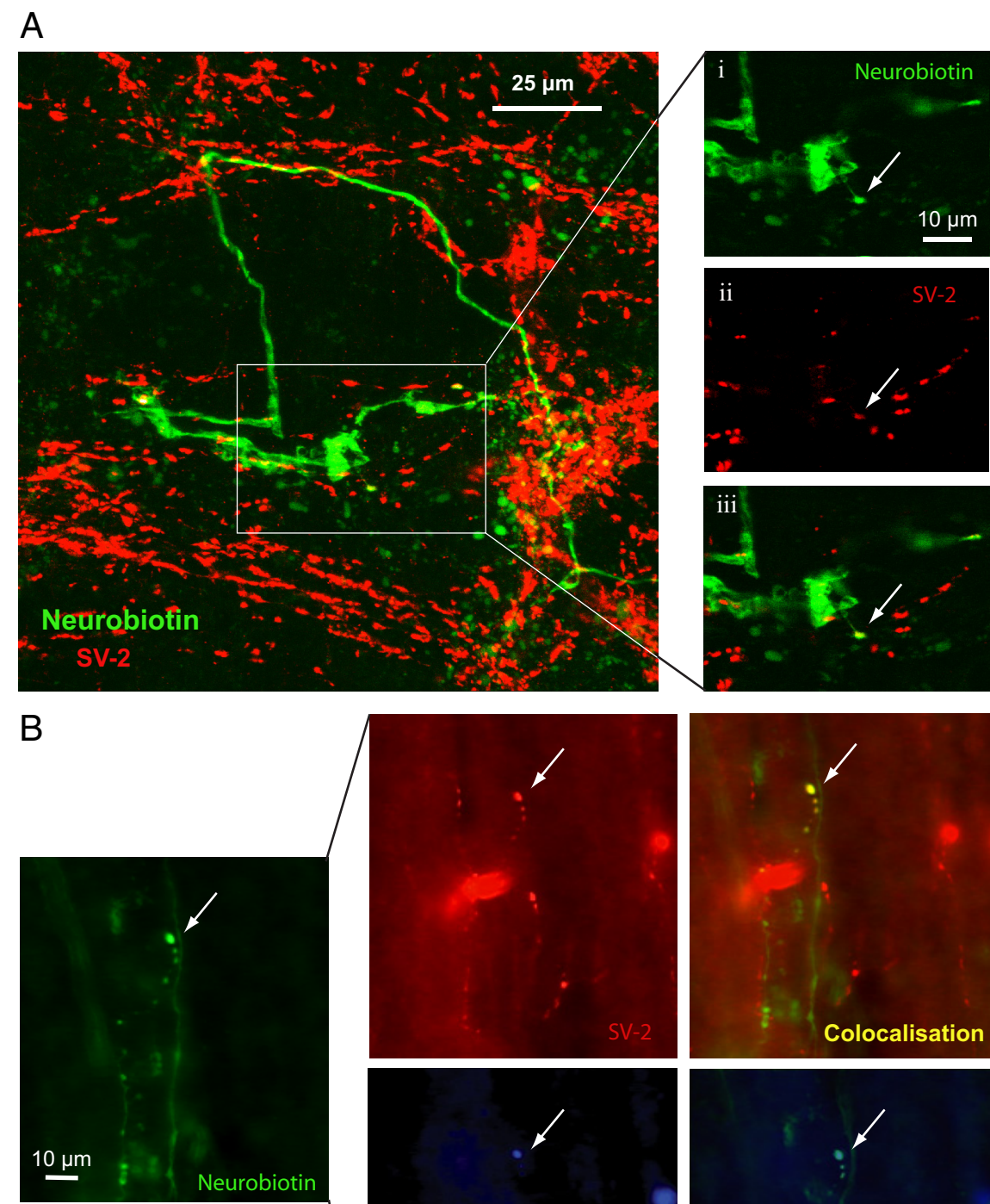

Figure 4. Synaptic vesicle-associated proteins in bouton-like structures on new dendrite-derived axon-like processes of axotomized motor neurons. A, Intracellular labeling using Neurobiotin (green) of an ALP with bouton-like structures and immunohistochemical labeling using an antibody specific for the SV-2 protein (red). Because of the density of SV-2 labeling in the gray matter, an example was selected from the white matter for clarity of illustration. The main figure is from a confocal stack of $40 \mathrm{X}$ $1-\mu \mathrm{m}$-thick optical slices. Inset $\boldsymbol{i}, 1-\mu \mathrm{m}$-thick confocal section showing a bouton-like structure (indicated with white arrow) intracellularly labeled with Neurobiotin (i), SV-2 labeling (ii), and with colocalization performed using ImageJ software (iii). $\boldsymbol{B}$, Another example of an ALP with bouton-like structures (indicated with white arrow) in the white matter. Immunohistochemical labeling using the SV-2 protein (red) colocalizes with the bouton-like structures (arrows). Immunohistochemical labeling using an antibody specific for the synaptophysin protein (blue) also colocalizes with the same bouton-like structures (arrows). The images in $\boldsymbol{B}$ were taken using an epifluorescent microscope and a $50-\mu \mathrm{m}$-thick tissue section, and colocalization was performed using

al., 2008). The observation that a single neuron develops more than one DDSA, however, may speak against this interpretation. Furthermore, in the current experiments, no DDSAs were observed traveling toward the ventral roots. In fact, no preferred trajectory was apparent, and the DDSAs traveled rostrally or caudally or dorsally before terminating $\geq 2 \mathrm{~mm}$ from the cell body. This would suggest that the potential targets of DDSAs may not be the same as the original axon and, thus, DDSAs do not replace the injured axon. One 
possibility is that DDSAs convert the motor neuron into a new type of interneuron combining the connections of the axon collaterals from the original axon with a new set of connections provided by the DDSAs. The function of these new connections is unclear, but they could provide a route to convey descending excitatory drive to other motor neurons that may not be axotomized. It is unknown what neurotransmitters are released by DDSAs, although they are immunoreactive for ChAT at 6 weeks post-axotomy (Hoang et al., 2005). However, it is known that axon collaterals of spinal motor neurons can corelease glutamate (to excite Renshaw cells and other motor neurons) and acetylcholine (Mentis et al., 2005; Nishimaru et al., 2005).

It is also plausible that DDSAs may play a role in the survival of the injured cell via the retrograde transport of neurotrophic factors such as brain-derived neurotrophic factor (BDNF). BDNF is retrogradely transported by axons of spinal motor neurons and exerts a neuroprotective effect on spinal motor neurons following axotomy, promoting cell survival and enhancing regeneration (Yan et al., 1992; Novikov et al., 1997). The usual source of BDNF is muscle. However, there are other sources of BDNF that are in the spinal cord, including primary afferents (Walker et al., 2001; Li et al., 2009), the motor neuron itself (Kobayashi et al., 1996; Johnson et al., 2000), and noradrenergic axons (Fawcett et al., 1998).

\section{Mechanisms underlying the formation of DDSAs}

Several studies have described the intracellular signal cascades responsible for the development and maintenance of neuronal polarity (Inagaki et al., 2001; Jiang et al., 2005; Yoshimura et al., 2005; Witte et al., 2008). Proximal axotomies may indirectly interfere with these cascades in vivo. Both upstream effectors in these signaling cascades, for example brain-derived neurotrophic factor and its receptor trkB, and downstream effectors, for example, CRMP-2 (collapsin response mediator protein 2), are upregulated following axotomy (Kobayashi et al., 1996; Suzuki et al., 2003).

Recent work has also suggested a crucial role for the AIS in the maintenance of neuronal polarity (Rasband, 2010). Loss of the AIS scaffolding protein ankyrin $G$ disrupts the axon initial segment causing axons to acquire the molecular and structural characteristics of dendrites (Hedstrom et al., 2008). Disruption of the axon initial segment has been demonstrated both in vitro and in vivo following ischemic injury and optic nerve crush (Schafer et al., 2009), and it has been hypothesized from these studies that disruption of the axon initial segment following peripheral nerve injury may explain other alterations in neuronal polarity in axotomized neurons such as we report here. However, in our injury model there is both anatomical and electrophysiological evidence that the original axon initial segment is functionally intact. All of the motoneurons in this study were antidromically activated, similar to previous studies at post-axotomy intervals as short as 2 weeks. Since DDSAs started to form at 2 weeks post-axotomy (albeit at a very immature stage) (see MacDermid et al., 2004), we cannot preclude a disruption of the initial segment at times before 2 weeks. However, at no time points studied thus far have we seen any indication of the original axon acquiring dendritic features, either morphological or molecular. These observations are, in fact, consistent with the concept of the axon initial segment as a physical/molecular barrier protecting the axonal identity of the axon by excluding dendritic components as has been suggested (Rasband, 2010). However, although disruption of the axon initial segment may be a prerequisite for the conversion of axons to dendrites, our results suggest that disruption of the AIS does not appear to be necessary for the conversion of dendrites to axons.

\section{Implications for the injured nervous system}

In the present study, axotomies were experimentally induced and specific measures were taken to prevent regeneration. This leads to the question: could DDSAs form in response to peripheral nerve damage as a consequence of traumatic injuries, such as damage to the brachial plexus? While it is well established that injured peripheral nerves have the capacity to regenerate, regeneration is rarely $100 \%$ successful and is often much less following proximal or more severe injuries (Krarup et al., 2002; Reyes et al., 2005). Thus, it is likely that a population of permanently axotomized motor neurons will be a common outcome of many types of proximal peripheral nerve injuries. It has also been demonstrated that following a midsagittal transection of the spinal cord, some distal dendritic processes of axotomized commissural spinal interneurons were immunoreactive for the axonal growth protein GAP-43 (Fenrich et al., 2007). These were only observed on cells whose axons failed to regenerate. Thus, the conversion of dendrites to axons may also occur following axotomy caused by spinal cord injuries. Given that DDSAs possess the molecular machinery necessary to function as axons, it is now vital to determine their synaptic connections, the intracellular signaling cascades responsible for their formation, and their ultimate fate.

\section{References}

Bannatyne BA, Edgley SA, Hammar I, Jankowska E, Maxwell DJ (2003) Networks of inhibitory and excitatory commissural interneurons mediating crossed reticulospinal actions. Eur J Neurosci 18:2273-2284.

Boiko T, Rasband MN, Levinson SR, Caldwell JH, Mandel G, Trimmer JS, Matthews G (2001) Compact myelin dictates the differential targeting of two sodium channel isoforms in the same axon. Neuron 30:91-104.

Boiko T, Van Wart A, Caldwell JH, Levinson SR, Trimmer JS, Matthews G (2003) Functional specialization of the axon initial segment by isoformspecific sodium channel targeting. J Neurosci 23:2306-2313.

Bradke F, Dotti CG (2000) Differentiated neurons retain the capacity to generate axons from dendrites. Curr Biol 10:1467-1470.

Craner MJ, Lo AC, Black JA, Waxman SG (2003) Abnormal sodium channel distribution in optic nerve axons in a model of inflammatory demyelination. Brain 126:1552-1561.

Cullheim S, Kellerth JO (1978) A morphological study of the axons and recurrent axon collaterals of cat sciatic alpha-motoneurons after intracellular staining with horseradish peroxidase. J Comp Neurol 178:537-557.

Fawcett JP, Bamji SX, Causing CG, Aloyz R, Ase AR, Reader TA, McLean JH, Miller FD (1998) Functional evidence that BDNF is an anterograde neuronal trophic factor in the CNS. J Neurosci 18:2808-2821.

Fenrich KK, Skelton N, MacDermid VE, Meehan CF, Armstrong S, NeuberHess MS, Rose PK (2007) Axonal regeneration and development of de novo axons from distal dendrites of adult feline commissural interneurons after a proximal axotomy. J Comp Neurol 502:1079-1097.

Finley MF, Kulkarni N, Huettner JE (1996) Synapse formation and establishment of neuronal polarity by P19 embryonic carcinoma cells and embryonic stem cells. J Neurosci 16:1056-1065.

Fletcher TL, Cameron P, De Camilli P, Banker G (1991) The distribution of synapsin I and synaptophysin in hippocampal neurons developing in culture. J Neurosci 11:1617-1626.

Gomis-Rüth S, Wierenga CJ, Bradke F (2008) Plasticity of polarization: changing dendrites into axons in neurons integrated in neuronal circuits. Curr Biol 18:992-1000.

Havton L, Kellerth JO (1987) Regeneration by supernumerary axons with synaptic terminals in spinal motoneurons of cats. Nature 325:711-714.

Hayashi K, Kawai-Hirai R, Ishikawa K, Takata K (2002) Reversal of neuronal polarity characterized by conversion of dendrites into axons in neonatal rat cortical neurons in vitro. Neuroscience 110:7-17.

Hedstrom KL, Rasband MN (2006) Intrinsic and extrinsic determinants of ion channel localization in neurons. J Neurochem 98:1345-1352.

Hedstrom KL, Ogawa Y, Rasband MN (2008) AnkyrinG is required for 
maintenance of the axon initial segment and neuronal polarity. J Cell Biol 183:635-640.

Hoang TX, Nieto JH, Havton LA (2005) Regenerating supernumerary axons are cholinergic and emerge from both autonomic and motor neurons in the rat spinal cord. Neuroscience 136:417-423.

Inagaki $\mathrm{N}$, Chihara $\mathrm{K}$, Arimura $\mathrm{N}$, Ménager $\mathrm{C}$, Kawano $\mathrm{Y}$, Matsuo N, Nishimura T, Amano M, Kaibuchi K (2001) CRMP-2 induces axons in cultured hippocampal neurons. Nat Neurosci 4:781-782.

Jiang H, Guo W, Liang X, Rao Y (2005) Both the establishment and the maintenance of neuronal polarity require active mechanisms: critical roles of GSK-3beta and its upstream regulators. Cell 120:123-135.

Johnson RA, Okragly AJ, Haak-Frendscho M, Mitchell GS (2000) Cervical dorsal rhizotomy increases brain-derived neurotrophic factor and neurotrophin-3 expression in the ventral spinal cord. J Neurosci 20:RC77(1-5).

Kaplan MR, Cho MH, Ullian EM, Isom LL, Levinson SR, Barres BA (2001) Differential control of clustering of the sodium channels $\mathrm{Na}(\mathrm{v}) 1.2$ and $\mathrm{Na}(\mathrm{v}) 1.6$ at developing CNS nodes of Ranvier. Neuron 30:105-119.

Kobayashi NR, Bedard AM, Hincke MT, Tetzlaff W (1996) Increased expression of BDNF and trkB mRNA in rat facial motoneurons after axotomy. Eur J Neurosci 8:1018-1029.

Kole MH, Ilschner SU, Kampa BM, Williams SR, Ruben PC, Stuart GJ (2008) Action potential generation requires a high sodium channel density in the axon initial segment. Nat Neurosci 11:178-186.

Krarup C, Archibald SJ, Madison RD (2002) Factors that influence peripheral nerve regeneration: an electrophysiological study of the monkey median nerve. Ann Neurol 51:69-81.

Li F, Li L, Song XY, Zhong JH, Luo XG, Xian CJ, Zhou XF (2009) Preconditioning selective ventral root injury promotes plasticity of ascending sensory neurons in the injured spinal cord of adult rats-possible roles of brain-derived neurotrophic factor, TrkB and $\mathrm{p} 75$ neurotrophin receptor. Eur J Neurosci 30:1280-1296.

Lindå H, Risling M, Cullheim S (1985) 'Dendraxons' in regenerating motoneurons in the cat: do dendrites generate new axons after central axotomy? Brain Res 358:329-333.

Lindå H, Cullheim S, Risling M (1992) A light and electron microscopic study of intracellularly HRP-labeled lumbar motoneurons after intramedullary axotomy in the adult cat. J Comp Neurol 318:188-208.

MacDermid V, Neuber-Hess M, Short C, Rose PK (2002) Alterations to neuronal polarity following permanent axotomy: a quantitative analysis of changes to MAP2a/b and GAP-43 distributions in axotomized motoneurons in the adult cat. J Comp Neurol 450:318-333.

MacDermid VE, Neuber-Hess MS, Rose PK (2004) The temporal sequence of morphological and molecular changes in axotomized feline motoneurons leading to the formation of axons from the ends of dendrites. J Comp Neurol 468:233-250.

Mentis GZ, Alvarez FJ, Bonnot A, Richards DS, Gonzalez-Forero D, Zerda R, O’Donovan MJ (2005) Noncholinergic excitatory actions of motoneurons in the neonatal mammalian spinal cord. Proc Natl Acad Sci U S A 102:7344-7349.

Nishimaru H, Restrepo CE, Ryge J, Yanagawa Y, Kiehn O (2005) Mammalian motor neurons corelease glutamate and acetylcholine at central synapses. Proc Natl Acad Sci U S A 102:5245-5249.

Novikov L, Novikova L, Kellerth JO (1997) Brain-derived neurotrophic fac- tor promotes axonal regeneration and long-term survival of adult rat spinal motoneurons in vivo. Neuroscience 79:765-774.

Quinn CC, Chen E, Kinjo TG, Kelly G, Bell AW, Elliott RC, McPherson PS, Hockfield S (2003) TUC-4b, a novel TUC family variant, regulates neurite outgrowth and associates with vesicles in the growth cone. J Neurosci 23:2815-2823.

Rasband MN (2010) The axon initial segment and the maintenance of neuronal polarity. Nat Rev Neurosci 11:552-562.

Reyes O, Sosa I, Kuffler DP (2005) Promoting neurological recovery following a traumatic peripheral nerve injury. P R Health Sci J 24:215-223.

Rose PK, Neuber-Hess M (1991) Morphology and frequency of axon terminals on the somata, proximal dendrites, and distal dendrites of dorsal neck motoneurons in the cat. J Comp Neurol 307:259-280.

Rose PK, Odlozinski M (1998) Expansion of the dendritic tree of motoneurons innervating neck muscles of the adult cat after permanent axotomy. J Comp Neurol 390:392-411.

Rose PK, MacDermid V, Joshi M, Neuber-Hess M (2001) Emergence of axons from distal dendrites of adult mammalian neurons following a permanent axotomy. Eur J Neurosci 13:1166-1176.

Schafer DP, Jha S, Liu F, Akella T, McCullough LD, Rasband MN (2009) Disruption of the axon initial segment cytoskeleton is a new mechanism for neuronal injury. J Neurosci 29:13242-13254.

Shrager P (1987) The distribution of sodium and potassium channels in single demyelinated axons of the frog. J Physiol 392:587-602.

Suzuki Y, Nakagomi S, Namikawa K, Kiryu-Seo S, Inagaki N, Kaibuchi K, Aizawa H, Kikuchi K, Kiyama H (2003) Collapsin response mediator protein-2 accelerates axon regeneration of nerve-injured motor neurons of rat. J Neurochem 86:1042-1050.

Takahashi D, Yu W, Baas PW, Kawai-Hirai R, Hayashi K (2007) Rearrangement of microtubule polarity orientation during conversion of dendrites to axons in cultured pyramidal neurons. Cell Motil Cytoskeleton 64:347-359.

Trimmer JS, Rhodes KJ (2004) Localization of voltage-gated ion channels in mammalian brain. Annu Rev Physiol 66:477-519.

Valtorta F, Pennuto M, Bonanomi D, Benfenati F (2004) Synaptophysin: leading actor or walk-on role in synaptic vesicle exocytosis? Bioessays 26:445-453.

Van Wart A, Trimmer JS, Matthews G (2007) Polarized distribution of ion channels within microdomains of the axon initial segment. J Comp Neurol 500:339-352.

Walker SM, Mitchell VA, White DM, Rush RA, Duggan AW (2001) Release of immunoreactive brain-derived neurotrophic factor in the spinal cord of the rat following sciatic nerve transection. Brain Res 899:240-247.

Westenbroek RE, Noebels JL, Catterall WA (1992) Elevated expression of type II $\mathrm{Na}+$ channels in hypomyelinated axons of shiverer mouse brain. J Neurosci 12:2259-2267.

Witte H, Neukirchen D, Bradke F (2008) Microtubule stabilization specifies initial neuronal polarization. J Cell Biol 180:619-632.

Yan Q, Elliott J, Snider WD (1992) Brain-derived neurotrophic factor rescues spinal motor neurons from axotomy-induced cell death. Nature 360:753-755.

Yoshimura T, Kawano Y, Arimura N, Kawabata S, Kikuchi A, Kaibuchi K (2005) GSK-3beta regulates phosphorylation of CRMP-2 and neuronal polarity. Cell 120:137-149. 